\title{
The elevated serum levels of calcineurin and nuclear factor of activated T-cells 1 in children with Kawasaki disease
}

\author{
Yameng Sun ${ }^{\dagger}$, Jingjing Liư ${ }^{\dagger}$ Zhimin Geng, Yijing Tao, Fenglei Zheng, Ying Wang, Songling Fu, Wei Wang, \\ Chunhong Xie, Yiying Zhang and Fangqi Gong ${ }^{*}$ (i)
}

\begin{abstract}
Background: The calcineurin and nuclear factor of activated T-cells (CaN-NFAT) signaling pathway had been found to be associated with Kawasaki disease (KD) susceptibility and coronary artery aneurysm formation as a contributor. To evaluate serum calcineurin (CaN) and nuclear factor of activated T-cells 1(NFAT1) levels in patients with Kawasaki disease (KD).

Methods: Serum levels of CaN and NFAT1 were measured by enzyme-linked immunosorbent assay method in 66 healthy children and $74 \mathrm{KD}$ patients at acute, afebrile and subacute stage.

Results: The serum levels of CaN and NFAT1 increased significantly in the acute stage, and decreased progressively in the afebrile and subacute stage, along with the reduction of C-reactive protein, white blood cells and neutrophil counts. And in the acute stage, the afebrile stage and the subacute stage, the expression of CaN and NFAT1 was upregulated significantly in KD patients compared to that in the healthy control. After the IVIG treatment, the serum levels of CaN and NFAT1 declined significantly in IVIG responders. However, the CaN and NTAT1 levels in the IVIG non-responders declined slowly. And in the afebrile stage, the NFAT1 levels were lower in KD patients with coronary artery lesions (CALs) $(268.82 \pm 11.96 \mathrm{ng} / \mathrm{ml})$ than those without CALs $(285.84 \pm 25.13 \mathrm{ng} / \mathrm{ml})$. However, the serum levels of CaN in KD patients with CALs had no significant difference with those in KD patients without CALs.

Conclusions: The specific regulation of CaN and NFAT1 serum levels in the course of KD was suggested that both of them were related in the development of KD.
\end{abstract}

Keywords: Kawasaki disease, Coronary artery lesions, Calcineurin, Nuclear factor of activated T-cells

\section{Background}

Kawasaki disease (KD) is a systemic vasculitis disease that affects small- and medium-sized vessels. The most notably cardiovascular complications caused by KD are coronary artery lesions (CALs). Approximately 20 to $25 \%$ of untreated KD patients develop CALs, and KD is now the most frequent cause of childhood acquired

\footnotetext{
* Correspondence: gongfangqi@zju.edu.cn

${ }^{\dagger}$ Yameng Sun and Jingjing Liu contributed equally to this work. Children's Hospital, Zhejiang University School of Medicine, No. 3333, Binsheng Road, Hangzhou 310052, People's Republic of China
}

heart disease in industrialized countries [1]. Timely treatment with intravenous immunoglobulin (IVIG) can reduce the incidence of coronary artery aneurysms to approximately 4\% [2]. Hence, early diagnosis of KD is extremely important and necessary. However, there are no pathognomonic laboratory parameters for diagnosis of KD. Therefore, to make out the etiology and pathogenesis of $\mathrm{KD}$, and specific biomarkers to diagnose $\mathrm{KD}$ would be valuable for preventing serious CALs [3].

The calcineurin and nuclear factor of activated T-cells (CaN-NFAT) pathway was first identified in $\mathrm{T}$ cells,

(c) The Author(s). 2020 Open Access This article is licensed under a Creative Commons Attribution 4.0 International License, which permits use, sharing, adaptation, distribution and reproduction in any medium or format, as long as you give appropriate credit to the original author(s) and the source, provide a link to the Creative Commons licence, and indicate if changes were made. The images or other third party material in this article are included in the article's Creative Commons licence, unless indicated otherwise in a credit line to the material. If material is not included in the article's Creative Commons licence and your intended use is not permitted by statutory regulation or exceeds the permitted use, you will need to obtain permission directly from the copyright holder. To view a copy of this licence, visit http://creativecommons.org/licenses/by/4.0/ The Creative Commons Public Domain Dedication waiver (http://creativecommons.org/publicdomain/zero/1.0/) applies to the data made available in this article, unless otherwise stated in a credit line to the data. 
where NFAT acts as a master regulator of lymphocyte proliferation, differentiation, and development [4, 5]. $\mathrm{CaN}$ is a calcium-activated protein phosphatase, which on activation triggers calcium signaling casades including upregulation of inflammatory cytokines like interleukin (IL)-6, IL-10, IL-12, tumor necrosis factor- $\alpha$ (TNF- $\alpha$ ) $[6,7]$. The elevated serum calcineurin levels has been found in the early onset of coronary artery disease and was associated with the polymorphism at the promoter region on PPP3R1(Protein Phosphatase 3, Regulatory Subunit B, Alpha) [6]. Previous studies have been confirmed that $\mathrm{CaN}$ and NFAT plays an important role in lymphocyte development, myocardial hypertrophy, heart failure and coronary artery diseases $[6,8]$. NFAT1 $(\mathrm{N}-$ FATp or NFATc2), one of the NFAT transcription factors family, was regulated by $\mathrm{Ca}^{2+}$-signaling pathway, with triggering a rapid rise of intracellular $\mathrm{Ca}^{2+}$, followed by the activation of phosphatase CaN, NFAT dephosphorylation which can be inhibited by the CaN inhibitors (CNIs) cyclosporin A and FK506, translocation into the nucleus and combination with specific DNA elements in the regulatory regions of target genes [4, 9]. The NFAT1 serum levels has been confirmed to increase significantly in RA patients when compared with control group [10].

Two functional SNPs in CASP3(Caspase 3) and ITPKC (inositol-trisphosphate 3-kinase $\mathrm{C}$ ) have been identified to be significantly associated with susceptibility to KD [11-13]. ITPKC regulates negatively T-cell and B-cell activation through the $\mathrm{Ca}^{2+} / \mathrm{NFAT}$ signaling pathway, and the $\mathrm{C}$ allele may contribute to increased signaling through this pathway, leading to immune hyper-reactivity in KD [12]. The risk allele substitution of CASP3 prevented NFAT from combinating to the DNA sequence surrounding the SNP, resulting in the reduction of CASP3 expression in immune effector cells and thus influencing susceptibility to KD [13]. Moreover, several clinical trials which studied the $\mathrm{CaN}$ inhibitors (CNIs, such as cyclosporine $\mathrm{A}$ and Tacrolimus) in the treatment of IVIGresistant KD patients have been found to be effective and well tolerated $[14,15]$. However, there are no relevant clinical trials about the serum levels of $\mathrm{CaN}$ and NFAT1 with the progression of KD. In order to explore the value of serum CaN and NFAT1 levels in the course of KD, we studied the CaN and NFAT1 sera levels in different stages of KD and healthy control.

\section{Materials and methods}

\section{Ethical statement}

The study was approved by the ethical committee of the Children's Hospital, Zhejiang University School of Medicine and was based on the institution's guidelines for human studies. This study conformed to the ethics guidelines of the 1975 Declaration of Helsinki. Written informed consent was obtained from each patient's parents.

\section{Patients}

In this study, we enrolled 74 fever children ( 52 boys and 22 girls) diagnosed with KD according to the diagnostic criteria established by the KD Research Committee [16] from November 2015 to March 2017. All patients were treated with IVIG at $1 \mathrm{~g} / \mathrm{kg} / \mathrm{d}$ for 2 days and oral aspirin at 30 to $50 \mathrm{mg} / \mathrm{kg} / \mathrm{d}$ at the Children's Hospital, Zhejiang University School of Medicine. After 3 to 5 days of treatment, while the patients' temperature had returned to normal range, the dosage of aspirin was reduced to 3 to $5 \mathrm{mg} / \mathrm{kg} /$ day for 8 to 12 weeks.

There were 9 IVIG-resistant KD patients (7 boys and 2 girls) who were with persistent or recrudescent fever $\left(>38^{\circ} \mathrm{C}\right)$ after $48 \mathrm{~h}$ standard treatment with IVIG, finally accepting another IVIG treatment or glucocorticoids. Nine patients including 7 boys and 2 girls were diagnosed with CALs including transiently dilated coronary arteries and aneurysms (defined as coronary artery zscore $>2$ ) [2] by 2-dimensional Echocardiography. Four patients had CALs in both the left and right coronary arteries, four in the left coronary artery, and one in the right coronary artery. The CALs complications in the seven patients were improved significantly and disappeared in 3 months after standard treatment. Two patients with CALs have persisted more than 9 and 12 months at the last follow-up. In addition, there were 66 healthy children were enrolled into the control group.

\section{Laboratory analysis}

Venous blood samples were respectively collected from the KD patients in three different stages. The acute stage was at the time from the disease diagnosed and admission, and before the IVIG treatment. The afebrile stage was at 3 days after the IVIG treatment and the patients' temperature had recovered to normal range. The subacute stage was about 14 to 21 days when the patients were first followed up after discharge. The control blood samples were collected when they came for the routine health examination.

Fresh venous blood samples were left to clot at room temperature for $30 \mathrm{~min}$ immediately following collection and then centrifuged at $3000 \mathrm{~g}$ for $10 \mathrm{~min}$. Then the serum samples were aliquoted and stored at $-80^{\circ} \mathrm{C}$ for further analysis. White blood cell (WBC) counts, neutrophil counts (N), platelet counts (PLT), Hemoglobin $(\mathrm{Hb})$, serum $\mathrm{C}$-reactive protein $(\mathrm{CRP})$, erythrocyte sedimentation rate (ESR), N-terminal pronatriuretic peptide (NT-ProBNP), procalcitonin (PCT), alanine aminotransferase (ALT), aspartate aminotransferase (AST), IL-2, IL4, IL-6, IL-10, TNF- $\alpha$ and Interferon- $\gamma$ (IFN- $\gamma$ ) were measured by conventional methods in our hospital 
laboratory. Serum levels of NFAT1 and CaN were quantified by using Enzyme-linked Immunosorbent Assay Kit For NFAT1 (ELISA Kit for Nuclear Factor Of Activated T-Cells, Cytoplasmic 2 (NFATc2); SEL942Hu, cloudclone corp. Houston, USA) and Calcineurin (CaN) (ELISA Kit for Calcineurin (CaN); SEB323Hu, cloudclone corp. Houston, USA). The limits of detection for the NFAT1 and CaN ELISA were $5.9 \mathrm{pg} / \mathrm{mL}$ and 0.057 $\mathrm{ng} / \mathrm{mL}$ respectively. The NFAT1 and CaN ELISA kit both show no cross-reactivity or interference with any of the cytokines tested.

\section{Statistical analysis}

All the data were analyzed using SPSS version 22.0 software and presented as mean \pm SD except those indicated. Statistical significance between the groups of patients was assessed by using analysis of variance (ANOVA) followed by the least significant difference (LSD) test for multiple comparisons of normal group. Mann-Whitney U test was used for nonparametric data. The differences were considered significant when the $p$-values were $<0.05$.

\section{Results}

Characteristics of the KD patients and healthy children

The demographic and clinical characteristics of these patients are shown in Table 1. In our study, there are 74
KD patients ( 22 girls and 52 boys) and 66 healthy children (33 boys and 33 girls) enrolled. The median age of KD patients was 26 months (range 3-119 months). There were no significant differences in age and weight between KD patients and healthy control. The duration of fever days was 2-15 days in KD patients. At the acute stage, WBC, N and CRP increased significantly, and then decreased with the course of the disease. Especially after the IVIG treatment, WBC, N and CRP decreased rapidly to the normal. However, at afebrile stage, PLT and ESR was much higher than those in acute stage significantly, and then, decreased gradually to the normal.

The serum levels of NFAT1 and CaN increased significantly at the acute stage of $\mathrm{KD}$ when compared with control group. At the afebrile stage and subacute stage, both NFAT1 and CaN decreased gradually with the process of $\mathrm{KD}$, but remained higher than that of the healthy control (Fig. 1).

\section{Serum levels of CaN and NFAT1 in IVIG responding and non-responding KD patients}

The serum levels of CaN and NFAT1 in IVIG responding and non-responding $\mathrm{KD}$ patients are shown in Table 2. In our study, there were $9 \mathrm{KD}$ patients (7 boys and 2 girls) who were not sensitive to the IVIG treatment. After the IVIG treatment, the serum levels of $\mathrm{CaN}$

Table 1 Characteristics of the patients with Kawasaki disease and Healthy control

\begin{tabular}{|c|c|c|c|c|c|c|c|}
\hline \multirow[t]{2}{*}{ Characteristics } & \multicolumn{6}{|l|}{ Kawasaki disease } & \multirow[t]{2}{*}{ Healthy Control } \\
\hline & Acute stage & $p$ value & Afebrile stage & $p$ value & Subacute stage & $p$ value & \\
\hline boys/girls & $52 / 22$ & 0.015 & & & & & $33 / 33$ \\
\hline Age, months (range) & 26(3-119) & 0.316 & & & & & $33(2-169)$ \\
\hline Weight (kg) & $14.7(6.5-43)$ & 0.416 & & & & & $14.8(6.8-47)$ \\
\hline Days of fever (range) & $5.5(2-15)$ & & & & & & - \\
\hline $\mathrm{CaN}(\mathrm{ng} / \mathrm{ml})$ & $2.97 \pm 0.63$ & $<0.001$ & $2.71 \pm 0.57^{* *}$ & $<0.001$ & $2.46 \pm 0.49^{* * \boldsymbol{\Lambda} \Delta}$ & $<0.001$ & $1.46 \pm 0.33$ \\
\hline NFAT1(pg/ml) & $305.62 \pm 29.29$ & $<0.001$ & $283.77 \pm 24.51^{* *}$ & $<0.001$ & $259.71 \pm 24.42^{* * \boldsymbol{\Delta} \Delta}$ & $<0.001$ & $218.08 \pm 32.10$ \\
\hline $\mathrm{WBC}\left(\times 10^{9} / \mathrm{L}\right)$ & $14.67 \pm 6.13$ & $<0.001$ & $8.5 \pm 3.38^{* *}$ & 0.355 & $7.53 \pm 2.09^{* *}$ & $<0.001$ & $8.9 \pm 2.36$ \\
\hline NFAT/WBC & $27.12 \pm 21.58$ & 0.756 & $38.64 \pm 15.55^{* *}$ & $<0.001$ & $37.03 \pm 11.08^{* *}$ & $<0.001$ & $26.27 \pm 8.99$ \\
\hline CaN/WBC & $0.27 \pm 0.24$ & 0.004 & $0.38 \pm 0.19^{* *}$ & $<0.001$ & $0.35 \pm 0.12^{*}$ & $<0.001$ & $0.18 \pm 0.07$ \\
\hline$N\left(\times 10^{9} / L\right)$ & $10.12 \pm 5.04$ & $<0.001$ & $3.76 \pm 2.65^{* *}$ & 0.821 & $2.78 \pm 1.34^{* *}$ & $<0.001$ & $3.85 \pm 1.82$ \\
\hline$N(\%)$ & $66 \pm 15$ & $<0.001$ & $41 \pm 16^{* *}$ & 0.644 & $36 \pm 13^{* *}$ & 0.012 & $42 \pm 13$ \\
\hline CRP (mg/l) & $75.26 \pm 52.59$ & $<0.001$ & $13.96 \pm 14.83^{* *}$ & $<0.001$ & $3.18 \pm 2.83^{* * \boldsymbol{\Lambda}}$ & 0.948 & $3.15 \pm 3.09$ \\
\hline $\operatorname{PLT}\left(\times 10^{9} / \mathrm{L}\right)$ & $371.23 \pm 147.02$ & 0.013 & $549.30 \pm 168.20^{* *}$ & $<0.001$ & $361.31 \pm 162.99 \Delta \mathbf{\Delta}$ & 0.003 & $320.98 \pm 70.44$ \\
\hline ESR (mm/h) & $71.89 \pm 33.75$ & - & $88.81 \pm 29.18^{* *}$ & - & $18.57 \pm 15.27^{* * \boldsymbol{\Lambda}}$ & & - \\
\hline IL-2(pg/ml) & $4.15 \pm 1.28$ & $<0.001$ & & & & & $3.05 \pm 0.89$ \\
\hline IL-4(pg/ml) & $5.31 \pm 5.00$ & $<0.001$ & & & & & $2.633 \pm 0.69$ \\
\hline IL-6(pg/ml) & $97.3 \pm 139.7$ & $<0.001$ & & & & & $11.7 \pm 19.87$ \\
\hline IL-10(pg/ml) & $17.76 \pm 21.03$ & $<0.001$ & & & & & $4.86 \pm 5.7$ \\
\hline
\end{tabular}

All $p$ values are for patients versus healthy control. CaN Calcineurin, NFAT nuclear factor of activated T cells, WBCs white blood cells, $N$ neutrophil, CRP C-reactive protein, PLT platelet, ESR erythrocyte sedimentation rate, IL-2 Interleukin-2; ${ }^{* *} p<0.01$ compared with acute stage; ${ }^{\boldsymbol{\Delta}} p<0.05,{ }^{\boldsymbol{\Delta}} p<0.01$ compared with afebrile stage 

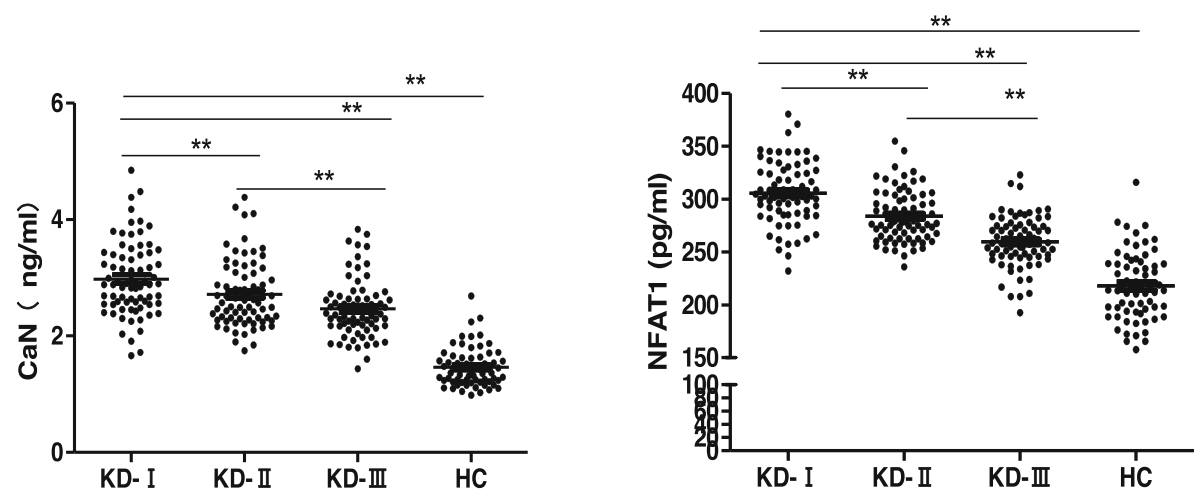

Fig. 1 The serum levels of CaN and NFAT1 in patients with KD and healthy control. KD-I: The acute stage; KD-II: The afebrile stage; KD-III: The subacute stage; HC: The healthy control. ${ }^{* *} p<0.01$

and NFAT1 declined significantly in IVIG responders. However, the CaN levels in the IVIG non-responders declined slowly, in which there were no significant differences between the acute, afebrile and subacute stages in IVIG non-responders KD patients. The same results can also be found in the NFAT levels of IVIG nonresponders KD patients when comparing the NFAT levels between the acute afebrile and subacute stages. Nevertheless, if comparing the CaN and NFAT1 levels between IVIG responder and non-responders KD patients, none of them showed significant differences in the acute, afebrile and subacute stages.

\section{Serum levels of CaN and NFAT1 in KD patients with or without CALs}

The serum levels of $\mathrm{CaN}$ and NFAT1 in KD patients with or without CALs are shown in Table 3. The results showed that the serum levels of CaN and NFAT1 descended significantly after treated with IVIG in KD patients with or without CALs. The serum levels of CaN had no significant difference between those KD patients with or without CALs. The serum levels of NFAT1 in KD patients with CALs were lower than those in KD patients without CALs at all of three stages. Especially at afebrile stage, the NFAT1 levels of KD patients with
CALs were significantly lower than those of KD patients without CALs $(268.82 \pm 11.96 \mathrm{pg} / \mathrm{ml}$ vs $285.84 \pm 25.13$ $\mathrm{pg} / \mathrm{ml}, P=0.003)$. In addition, we found that IL-4, IL-6 and IL-10 levels in KD patients with CALs were much lower than that in KD patients without CALs.

\section{Serum levels of CaN per WBC at different stages of KD patients}

In order to figure out whether the $\mathrm{CaN}$ serum level was due to the prominent increasing WBC. We compared the CaN level by standardizing to WBC (Table 1 and Fig. 2). The results showed that the CaN level per WBC was lower in acute stage of KD than that in afebrile and subacute stage. When compared with healthy control, the CaN level per WBC in acute stage was much higher. In the afebrile and subacute stage, along with the recovery of inflammation, the WBC decreased rapidly, but the CaN level didn't decrease synchronously. Therefore, the CaN serum level per WBC was much higher than that in the acute stage of KD and the healthy control.

\section{Discussion}

According to our study, in the acute stage of $K D$, the serum levels of CaN and NFAT1 increased to peak with the course of disease. After the treatment of IVIG, the

Table 2 Serum levels of CaN and NFAT1 in IVIG responding and non-responding patients with KD

\begin{tabular}{|c|c|c|c|c|}
\hline Characteristics & stage & IVIG responders $(n=65)$ & IVIG non-responders $(n=9)$ & $p$ value \\
\hline Boys/girls & & $45 / 20$ & $7 / 2$ & 0.716 \\
\hline \multirow[t]{3}{*}{$\mathrm{CaN}(\mathrm{ng} / \mathrm{ml})$} & Acute stage & $3.02 \pm 0.64$ & $2.67 \pm 0.50$ & 0.125 \\
\hline & Afebrile stage & $2.74 \pm 0.57^{\wedge}$ & $2.48 \pm 0.48$ & 0.163 \\
\hline & Subacute stage & $2.47 \pm 0.49^{\boldsymbol{\Delta} *}$ & $2.34 \pm 0.54$ & 0.354 \\
\hline \multirow[t]{3}{*}{$\operatorname{NFAT1}(\mathrm{pg} / \mathrm{ml})$} & Acute stage & $304.35 \pm 29.35$ & $314.84 \pm 28.76$ & 0.317 \\
\hline & Afebrile stage & $283.26 \pm 24.81^{\boldsymbol{\Lambda} \Lambda}$ & $287.94 \pm 22.98$ & 0.726 \\
\hline & Subacute stage & $258.43 \pm 24.32^{\boldsymbol{\Delta} \boldsymbol{\Delta} * *}$ & $270.27 \pm 24.09 \boldsymbol{\Lambda}$ & 0.145 \\
\hline
\end{tabular}

$\mathbf{\Delta}_{p}<0.05,{ }^{\wedge} \boldsymbol{\Delta} p<0.01$ compared with acute stage. ${ }^{*} p<0.05,{ }^{* *} p<0.01$ compared with afebrile stage 
Table 3 Serum levels of CaN and NFAT1 in Kawasaki disease with or without coronary artery lesions

\begin{tabular}{|c|c|c|c|c|}
\hline Characteristics & stage & KD with CALs $(n=9)$ & KD without CALs $(n=65)$ & $p$ value \\
\hline Boys/girls & & $7 / 2$ & $45 / 20$ & 0.716 \\
\hline \multirow[t]{3}{*}{$\mathrm{CaN}(\mathrm{ng} / \mathrm{ml})$} & Acute stage & $3.13 \pm 0.52$ & $2.95 \pm 0.65$ & 0.444 \\
\hline & Afebrile stage & $2.77 \pm 0.46$ & $2.70 \pm 0.58^{\wedge}$ & 0.743 \\
\hline & Subacute stage & $2.50 \pm 0.44^{\wedge}$ & $2.45 \pm 0.50^{* \boldsymbol{\Lambda} \boldsymbol{\Lambda}}$ & 0.226 \\
\hline \multirow[t]{3}{*}{$\operatorname{NFAT1}(\mathrm{pg} / \mathrm{ml})$} & Acute stage & $297.46 \pm 13.84$ & $306.75 \pm 30.73$ & 0.135 \\
\hline & Afebrile stage & $268.82 \pm 11.96^{\mathbf{\Lambda}}$ & $285.84 \pm 25.13^{\mathbf{\Lambda}}$ & 0.003 \\
\hline & Subacute stage & $250.43 \pm 20.15^{* \Delta \wedge}$ & $261.00 \pm 24.81^{* * \boldsymbol{\Delta} \Delta}$ & 0.226 \\
\hline |L-2(pg/ml) & Acute stage & $3.83 \pm 1.28$ & $4.20 \pm 1.28$ & 0.442 \\
\hline IL-4(pg/ml) & Acute stage & $3.5 \pm 1.15$ & $5.61 \pm 5.33$ & 0.013 \\
\hline IL-6(pg/ml) & Acute stage & $35.19 \pm 29.14$ & $107.8 \pm 148.28$ & 0.002 \\
\hline IL-10(pg/ml) & Acute stage & $8.30 \pm 6.99$ & $19.36 \pm 22.21$ & 0.006 \\
\hline
\end{tabular}

$\mathbf{\Delta} P<0.05, \mathbf{\Lambda} P<0.01$ compared with patients at acute stage

${ }^{*} P<0.05,{ }^{*} P<0.01$ compared with patients at afebrile stage

$\mathrm{CaN}$ and NFAT1 levels decreased gradually at the afebrile stage and subacute stage, along with the reduction of CRP, WBC and neutrophils. The serum levels of $\mathrm{CaN}$ and NFAT1 at the subacute stage were still significantly higher than those in the healthy control, which means that the CaN and NFAT1 levels didn't return to normal at the subacute stage. Our data indicated that $\mathrm{CaN}$ and NFAT1 signal pathway could play a potential role in the pathophysiology of KD. In addition, it suggests that the reduction of inflammation is associated with the reduction of NFAT1 sera levels. By using the CNIs to block the NFAT translocation to the nucleus, the inflammation of KD at acute stage could be relieved.

The CaN-NFAT signal pathway as a contributor had been found to be associated with KD susceptibility and CALs formation, growing out of 3 functional single nucleotide polymorphisms (SNPs) in ITPKC, CASP3 and SLC8A1(solute carrier family 8, member 1 , a sodium/ calcium exchanger encoding NCX1) gene [11, 12, 17].

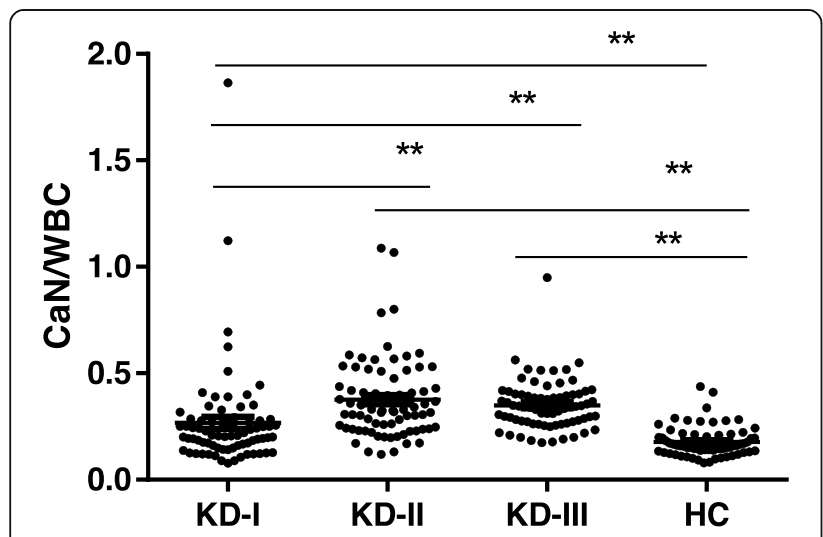

Fig. 2 CaN level per WBC at different stage of KD treated with intravenous immunoglobulin. KD-I, acute stage; KD-II, afebrile stage; KD-III, subacute stage; HC, healthy control. ${ }^{* *} p<0.001$
Previous studies had demonstrated that $\mathrm{T}$ effector memory cells with a proinflammatory phenotype and macrophages were involved in the acute stage of $\mathrm{KD}$, and pathology findings of coronary artery autopsy tissues showed that the coronary arterial wall was infiltrated by CD8+ T cells $[18,19]$. The flow cytometry results in the IVIG-responsiveness KD showed that extreme CD8 + T cell activation and an disequilibrium in $\mathrm{CD} 8+\mathrm{T}$ cell activation and inhibition were important in the pathogenesis of KD [20]. The CaN-NFAT signal pathway had been confirmed to participate in the T-helper-cell differentiation, T-cell activation and T-cell tolerance, but also participate in the transcriptional program of CD8+ T cell exhaustion $[5,9,21]$. NFAT1 can cooperate with AP-1 to induce the expression of inflammatory cytokines $[9,21]$. Moreover, NFAT1 controls the exhaustion of CD8+ T cell by inducing the inhibitory surface receptors when not cooperate with AP-1 [21]. These researches may suggest that KD susceptibility, CALs formation and response to IVIG are associated with CaN-NFAT signaling pathway.

$\mathrm{CaN}$ is a key phosphatase in immunity, many of which are facilitated by dephosphorylation of NFAT and translocation of NFAT from the cytoplasm to the nucleus to regulate cell proliferation, differentiation and development [22]. In our study, the serum levels of $\mathrm{CaN}$ were significantly increased in the acute stage of $\mathrm{KD}$, coincident with the NFAT1 levels, which suggests that the pathway is activated. Once the CaN-NFAT pathway is activated, the NFAT is dephosphorylated and translocated to the nucleus, which modify immune cell maturation, cytokine production and release such as IL-2, IL-4, IL-6 and cell survival [5]. In our study, we also found the serum of IL-2, IL-4, IL-6, IL-10 were increasing at acute stage of KD when compared with healthy control. Previous studies have reported that in the early stage of KD, the TNF, IL-1 
and IL-6 signaling pathways were activated and the activated circulating neutrophils increased significantly, which is suggested the abnormal activation of innate immunity [23]. Moreover, recent studies have shown that CaNNFAT signaling also plays a vital role in innate cell activation, including the activation of dendritic cells (DCs) and natural killer cells (NK cells) which is primed by DCs and enhanced by DCs production of IL-2 [5]. Besides, CaNNFAT signaling regulated the expression of IL-6, IL-10, IL-12 and TNF- $\alpha$ in macrophages, most of which have been confirmed to be involved in the pathogenesis of KD [24-27]. On the other hand, in the first week of KD onset, proinflammatory and regulatory $\mathrm{T}$ cells are found in the circulation, which suggests the activation of adaptive immunity [19]. After the treatment of IVIG, the increase of the regulatory $\mathrm{T}$-cell population in the peripheral blood is related with defervescence and clinical improvement [28]. In our data, the levels of CaN and NFAT1 decreased significantly at the afebrile and subacute stage after the IVIG treatment, suggesting that the CaN-NFAT signaling pathway plays a potential role in onset of $\mathrm{KD}$, and along with the reduction of CaN and NFAT1 sera levels, the fever is resolved and inflammation is alleviated.

Although the serum levels of $\mathrm{CaN}$ in KD patients with CALs had no significant difference from those in KD patients without CALs due to limited sample size, the serum levels of NFAT1 in KD patients with CALs were lower significantly than those in KD patients without CALs at afebrile stage, which suggest that NFAT1 levels decline much rapidly after the standard IVIG treatment in the KD patients with CALs than those without CALs. A recent study has confirmed that homozygous KD patients for the SLC8A1 A (risk) allele of rs13017968 were more likely to develop coronary artery aneurysms [17], which suggested the activation and importance of calcium signaling pathway in KD patients with CALs. These findings seemed to be consistent with our study, suggesting the availability of using CNIs in KD with CALs. Blocking the calcium signaling pathway by CNIs, resulting in blocking the dephosphorylation of the transcription factor NFAT may reduce acute inflammation and CALs in KD patients.

According to our data, after the IVIG treatment, the serum levels of CaN and NFAT1 declined significantly in IVIG responders. However, the CaN and NTAT1 levels in the IVIG non-responders declined slowly, suggesting that the CaN and NFAT1 levels in IVIG resisitant KD patients cannot be decreased effectively by using IVIG. Furthermore, we found that at the acute and subacute stage, the serum levels of NFAT1 were little higher in the IVIG non-responders than those in IVIG responders (although there were no significant differences with the limited sample size), which suggest that the immune response in IVIG non-responders may be more severe than in IVIG-responders. Clinical trials have confirmed that $\mathrm{CaN}$ inhibitors are effective and have no serious adverse effects in the treatment of IVIG-resistant $\mathrm{KD}$ patients $[14,15]$. A recent study which is a randomised controlled, open-label, blinded-endpoints, phase 3 trial aiming to compare the efficacy of IVIG plus CNIs or IVIG alone when treating the KD patients with high risks for IVIG resistance, reported that combination therapy with IVIG and CsA can decrease the incidence of CALs [29]. Therefore, the mechanisms of CaN inhibitors in treating KD patients especially in IVIG resistance patients may be because of rapid defervescence and resolution of inflammation by specifically blocking the abnormal activation of CaN-NFAT pathway, sequentially suppressing the undesired innate immune and adaptive immune responses, including inhibiting the production of proinflammatory cytokines and a reduction of circulating activated $\mathrm{CD} 4+$ and $\mathrm{CD} 8+\mathrm{T}$ effector memory cells.

Previous studies have reported the WBC and activated circulating neutrophils increased significantly and also the apoptosis of circulating neutrophils in acute stage of $\mathrm{KD}$ was delayed [30]. Therefore, there is a hypothesis which needs to be confirmed-whether the elevated $\mathrm{CaN}$ and NFAT1 serum levels was caused by high WBC. As is known to all, NFAT2 and NFAT4 not NFAT1 were present in neutrophil, which is suggested that the increased NFAT1 level was not caused by neutrophil. In addition, previous study has reported that there were no significant increase in mononuclear cells between KD and control groups or in acute and convalescent stage of $\mathrm{KD}$ [23], which is suggesting the reason causing the increasing WBC in KD was the increasing Neutrophils. However, the subgroup of lymphocytes and monocytes/ macrophages were differentially regulated in acute stage of KD. Taken together, it is indicated that not the increased amount of WBC but the differential regulation of lymphocytes and monocytes/macrophages subgroups caused the increase of NFAT1 serum levels.

$\mathrm{CaN}$ as a heterodimeric serine/threonine phosphatase enzyme can be found in all tissues except $\gamma$ isoform [22] Thus, we compared the CaN level by standardizing to WBC. According to our results, the CaN level increased in the acute stage and gradually declined after treatment by IVIG. The WBC rised rapidly at acute stage with the delayed neutrophils apoptosis and declined rapidly with the promotion of neutrophils apoptosis due to the IVIG treatment. The $\mathrm{CaN}$ level per WBC were much higher than that in the acute stage of KD and the healthy control. The relative non-synchronization with WBC as they evolve from acute to chronic stage suggested that the elevated CaN serum level was not caused by high WBC. However, previous studies have reported that NFAT was involved in the regulation of apoptosis [31]. According 
to our results, after IVIG treatment, the NFAT1 serum levels shifted from high to close to normal. At the same time, after IVIG treatment, the apoptosis of neutrophils shifted from delayed to be promoted, appearing to be the same trend with the NFAT1 levels. Cytokines such as IL-1 $\beta$, IL-6, IFN- $\gamma$ and TNF-a which can be regulated by $\mathrm{CaN} / \mathrm{NFAT}$ signaling pathway can inhibit neutrophil apoptosis [30]. Whether the elvated NFAT1 in acute stage of KD resulted in delayed neutrophils apoptosis need to be further confirmed.

\section{Conclusions}

In conclusion, CaN and NFAT1 are both increased at the acute stage of $\mathrm{KD}$, and declined gradually at the afebrile stage and subacute stage after the treatment of IVIG, along with the resolution of inflammatory and clinical improvement. The specific regulation of $\mathrm{CaN}$ and NFAT1 serum levels in the course of KD was suggested that both of them were related in the development of KD.

\begin{abstract}
Abbreviations
ANOVA: Analysis of variance; CaN: Calcineurin; CASP3: Caspase-3; CNIs: CaN inhibitors; CRP: C-reactive protein; DCs: Dendritic cells; ELISA: Enzyme-linked immunosorbent assay; ESR: Erythrocyte sedimentation rate; $\mathrm{Hb}$ : Hemoglobin; IFN-ү: Interferon- $\gamma$; IL-6: Interleukin-6; ITPKC: Inositol 1,4,5-trisphosphate 3kinase C; IVIG: Intravenous immunoglobulin; KD: Kawasaki disease; LSD: Least significant difference; N: Neutrophil counts; $\mathrm{NCX} 1 \mathrm{Na}^{+}-\mathrm{Ca}^{2+}$ exchanger isoform 1; NFAT1: Nuclear factor of activated T-cells 1; NK cells : Natural killer cells; PLT: Platelet counts; SLC8A1: Solute carrier family 8, member 1; SNPs: Single nucleotide polymorphisms; TNF-a: Tumor necrosis factor-a; WBC: White blood cell
\end{abstract}

\section{Acknowledgements}

Not applicable.

\section{Authors' contributions \\ Yameng Sun, Jingjing Liu: Dr. Sun and Dr. Liu conceptualized and designed the study, acquired, analyzed and interpreted data, drafted the initial manuscript. Zhimin Geng, Yijing Tao, Fenglei Zheng, Ying Wang, Songling Fu, Wei Wang, Chunhong Xie, Yiying Zhang: Dr. Geng, Dr. Tao, Dr. Zheng, Dr. Wang, Dr. Fu, Dr. Wang, Dr. Xie and Dr. Zhang acquired, analyzed and interpreted data. Fangqi Gong: Dr. Gong conceptualized and designed the study, coordinated and supervised data collection and anylisis, critically reviewed the manuscript. All authors approved the final manuscript as submitted and agree to be accountable for all aspects of the work.}

\section{Funding}

This work is supported, in part, by grants from The National Natural Science Foundation of China (81670251).

\section{Availability of data and materials}

Data can be requested from the corresponding author.

\section{Ethics approval and consent to participate}

The study was approved by the medical ethics committee of the Children's Hospital, Zhejiang University School of Medicine and was based on the institution's guidelines for human studies. All patients gave signed informed consent.

\section{Consent for publication}

All authors consented to publication of the manuscript.

\section{Competing interests}

The authors declare that they have no competing interests.
Received: 31 January 2020 Accepted: 2 March 2020

Published online: 17 March 2020

\section{References}

1. Kobayashi T, Saji T, Otani T, Takeuchi K, Nakamura T, Arakawa H, et al. Efficacy of immunoglobulin plus prednisolone for prevention of coronary artery abnormalities in severe Kawasaki disease (RAISE study): a randomised, open-label, blinded-endpoints trial. Lancet. 2012; 379(9826):1613-20.

2. McCrindle BW, Rowley AH, Newburger JW, Burns JC, Bolger AF, Gewitz M, et al. Diagnosis, treatment, and long-term Management of Kawasaki Disease: a scientific statement for health professionals from the American Heart Association. Circulation. 2017;135(17):e927-99.

3. Ko TM, Kuo HC, Chang JS, Chen SP, Liu YM, Chen HW, et al. CXCL10/ IP-10 is a biomarker and mediator for Kawasaki disease. Circ Res. 2015; 116(5):876-83.

4. Rudolf R, Busch R, Patra AK, Muhammad K, Avots A, Andrau JC, et al. Architecture and expression of the nfatc1 gene in lymphocytes. Front Immunol. 2014;5:21.

5. Fric J, Zelante T, Wong AY, Mertes A, Yu HB, Ricciardi-Castagnoli P. NFAT control of innate immunity. Blood. 2012;120(7):1380-9.

6. Maddhuri S, Bandaru S, Bhukya C, Cingeetham V, Malempati AR, Deepika MLN, et al. Association of CnB 51/5D promoter gene polymorphism and serum calcineurin levels in early onset of coronary artery disease of south Indian cohort. Gene. 2017;632:1-6.

7. Parra V, Rothermel BA. Calcineurin signaling in the heart: the importance of time and place. J Mol Cell Cardiol. 2017;103:121-36.

8. Tham YK, Bernardo BC, Ooi JYY, Weeks KL, Mcmullen JR. Pathophysiology of cardiac hypertrophy and heart failure: signaling pathways and novel therapeutic targets. Arch Toxicol. 2015;89(9):1401-38.

9. Macian F. NFAT proteins: key regulators of T-cell development and function. Nat Rev Immunol. 2005;5(6):472-84.

10. Paradowska-Gorycka A, Romanowska-Prochnicka K, Haladyj E, Manczak M, Maslinski S, Olesinska M. Association of the Smad3 and NFATc2 gene polymorphisms and their serum levels with susceptibility to rheumatoid arthritis in polish cohorts. Clin Exp Immunol. 2015;179(3):444-53.

11. Onouchi Y, Suzuki Y, Suzuki H, Terai M, Yasukawa K, Hamada H, et al. ITPKC and CASP3 polymorphisms and risks for IVIG unresponsiveness and coronary artery lesion formation in Kawasaki disease. Pharmacogenomics J. 2013;13(1):52-9.

12. Onouchi Y, Gunji T, Burns JC, Shimizu C, Newburger JW, Yashiro M, et al. ITPKC functional polymorphism associated with Kawasaki disease susceptibility and formation of coronary artery aneurysms. Nat Genet. 2008; 40(1):35-42.

13. Onouchi Y, Ozaki K, Buns JC, Shimizu C, Hamada H, Honda T, et al. Common variants in CASP3 confer susceptibility to Kawasaki disease. Hum Mol Genet. 2010;19(14):2898-906.

14. Suzuki $H$, Terai M, Hamada $H$, Honda T, Suenaga T, Takeuchi T, et al. Cyclosporin a treatment for Kawasaki disease refractory to initial and additional intravenous immunoglobulin. Pediatr Infect Dis J. 2011;30(10): 871-6.

15. Tremoulet AH, Pancoast P, Franco A, Bujold M, Shimizu C, Onouchi Y, et al. Calcineurin inhibitor treatment of intravenous immunoglobulin-resistant Kawasaki disease. J Pediatr. 2012:161(3):506-512.e501.

16. Ayusawa M, Sonobe T, Uemura S, Ogawa S, Nakamura Y, Kiyosawa N, et al. Revision of diagnostic guidelines for Kawasaki disease (the 5th revised edition). Pediatr Int. 2005;47(2):232-4.

17. Shimizu C, Eleftherohorinou H, Wright VJ, Kim J, Alphonse MP, Perry JC, et al. Genetic variation in the SLC8A1 calcium signaling pathway is associated with susceptibility to Kawasaki disease and coronary artery abnormalities. Circ Cardiovasc Genet. 2016;9(6):559-68.

18. Brown TJ, Crawford SE, Cornwall ML, Garcia F, Shulman ST, Rowley AH. CD8 T lymphocytes and macrophages infiltrate coronary artery aneurysms in acute Kawasaki disease. J Infect Dis. 2001;184(7):940-3.

19. Franco A, Shimizu C, Tremoulet AH, Burns JC. Memory T-cells and characterization of peripheral T-cell clones in acute Kawasaki disease. Autoimmunity. 2010;43(4):317-24.

20. Ye Q, Gong FQ, Shang SQ, Hu J. Intravenous immunoglobulin treatment responsiveness depends on the degree of $\mathrm{CD} 8+\mathrm{T}$ cell activation in Kawasaki disease. Clin Immunol (Orlando, Fla). 2016;171:25-31. 
21. Martinez GJ, Pereira RM, Aijo T, Kim EY, Marangoni F, Pipkin ME, et al. The transcription factor NFAT promotes exhaustion of activated CD8(+) T cells. Immunity. 2015;42(2):265-78.

22. Musson RE, Cobbaert CM, Smit NP. Molecular diagnostics of calcineurinrelated pathologies. Clin Chem. 2012;58(3):511-22.

23. Matsubara T, Ichiyama T, Furukawa S. Immunological profile of peripheral blood lymphocytes and monocytes/macrophages in Kawasaki disease. Clin Exp Immunol. 2005;141(3):381-7.

24. Elloumi HZ, Maharshak N, Rao KN, Kobayashi T, Ryu HS, Muhlbauer M, et al. A cell permeable peptide inhibitor of NFAT inhibits macrophage cytokine expression and ameliorates experimental colitis. PLoS One. 2012;7(3):e34172.

25. Granucci F, Zanoni I, Ricciardicastagnoli P. Natural killer (NK) cell functions can be strongly boosted by activated dendritic cells (DC). Eur J Immunol. 2006;36(10):2819-20.

26. Liu Z, Lee J, Krummey S, Lu W, Cai H, Lenardo MJ. The kinase LRRK2 is a regulator of the transcription factor NFAT that modulates the severity of inflammatory bowel disease. Nat Immunol. 2011:12(11):1063-70.

27. Goodridge HS, Simmons RM, Underhill DM. Dectin-1 stimulation by Candida albicans yeast or zymosan triggers NFAT activation in macrophages and dendritic cells. J Immunol (Baltimore, Md : 1950). 2007:178(5):3107-15.

28. Franco A, Touma R, Song Y, Shimizu C, Tremoulet AH, Kanegaye JT, et al. Specificity of regulatory $T$ cells that modulate vascular inflammation. Autoimmunity. 2014;47(2):95-104

29. Hamada H, Suzuki H, Onouchi Y, Ebata R, Terai M, Fuse S, et al. Efficacy of primary treatment with immunoglobulin plus ciclosporin for prevention of coronary artery abnormalities in patients with Kawasaki disease predicted to be at increased risk of non-response to intravenous immunoglobulin (KAICA): a randomised controlled, open-label, blinded-endpoints, phase 3 trial. Lancet. 2019;393(10176):1128-37.

30. Tsujimoto H, Takeshita S, Nakatani K, Kawamura Y, Tokutomi T, Sekine I. Delayed apoptosis of circulating neutrophils in Kawasaki disease. Clin Exp Immunol. 2001;126(2):355-64.

31. Mognol GP, Carneiro FR, Robbs BK, Faget DV, Viola JP. Cell cycle and apoptosis regulation by NFAT transcription factors: new roles for an old player. Cell Death Dis. 2016;7:e2199.

\section{Publisher's Note}

Springer Nature remains neutral with regard to jurisdictional claims in published maps and institutional affiliations.

Ready to submit your research? Choose BMC and benefit from:

- fast, convenient online submission

- thorough peer review by experienced researchers in your field

- rapid publication on acceptance

- support for research data, including large and complex data types

- gold Open Access which fosters wider collaboration and increased citations

- maximum visibility for your research: over $100 \mathrm{M}$ website views per year

At $\mathrm{BMC}$, research is always in progress.

Learn more biomedcentral.com/submissions 